\title{
New doctor-patient communication learning software to help interns succeed in communication skills
}

\author{
Chao Sun ${ }^{1 \dagger}$, Junkai Zou², Lanbo Zhao', Qing Wang ${ }^{1}$, Shaozhi Zhang ${ }^{3}$, Qurat Ulain ${ }^{1}$, Qing Song ${ }^{1,4}$ and Qiling Li ${ }^{1 *}$ (D
}

\begin{abstract}
Background: Nowadays, the research of doctor-patient communication is becoming increasingly important not only in China but also around the world.

Methods: The study designs a type of learning software to train the interns to advance their communication skills, and whose validity for improving doctor-patient communication in self-controlled trials is evaluated. With the aid of the new learning software, the self-controlled tests were carried out among 183 interns to assess the quality of their communication skill acquisition. The learning effectiveness of the preparation stage, information collection, information given, patient understanding, and inquisition ending was evaluated with the Set Elicit Give Understand End (SEGUE) framework after 3 months of training.

Results: More interns (37.16\% vs. $10.98 \%, P<0.001)$ could accurately identify the psychosocial or emotional factors contributing to the diseases. An increased number of interns ( $42.62 \%$ vs. $10.40 \%, P<0.001)$ were able to openly discuss lifestyle issues and prevention strategies with patients. The study also revealed that interns who had completed training tended to allow patients more time to describe their feelings and concerns about their illnesses. In addition, more of the trained interns roved capable of being caring and respectful to patients and showing empathetic communication behavior (53.01\% vs. $26.59 \%, P<0.001)$.
\end{abstract}

Conclusions: The doctor-patient communication software may help the interns known more about communication skills.

Keywords: Doctor-patient, Communication, Learning software, Communication ability, Interns

\section{Background}

The doctor-patient relationship has become a complex social issue in China due to a lack of trust and mutual understanding between doctors and patients. As the Chinese Medical Doctors' Association reported, the number of violent attacks on doctors rose from 57 cases to 130 cases between 2010 and 2013 [1]. More than two-thirds of doctors surveyed admitted that tension and conflict exist between doctors and patients.

One study has indicated that $98.47 \%$ of hospitals were troubled by medical disputes, $70 \%$ of which were caused by ineffective communication between doctors

\footnotetext{
* Correspondence: liqiling@mail.xjtu.edu.cn

${ }^{\dagger}$ Chao Sun and Junkai Zou contributed equally to this work.

${ }^{1}$ First Affiliated Hospital, Xi'an Jiaotong University, 277 Yanta West Road, Xi'an, Shaanxi, China

Full list of author information is available at the end of the article
}

and patients [2]. The doctor-patient relationship in China can be described as patriarchal and is characterized by a doctor-centered mode of communication [3]. Doctors are expected to be the experts who give patients appropriate medical instruction as to what to do. Patient-centered interviewing is unusual in China. Patient-centered communication between doctors and patients is essential for the delivery of high-quality patient care [4]. Unlike other countries, a dilemma is often faced by the Chinese doctors [5]. Most Chinese families ask physicians not to reveal the diagnosis and prognosis to patients. However, more and more patients want to be fully informed, and the right of being informed has been regulated by law. Competent communication by doctors can improve health outcomes, enhance patient satisfaction, and contribute to 
doctors' job satisfaction [6]. Conversely, poor communication and attitude between doctor and patient may constitute the most frequent underlying cause of malpractice litigation, complaints against doctors, and nonadherence to medication regimens $[7,8]$. Therefore, the development of effective communication skills is an important part of becoming a good doctor. There is strong evidence that appropriate teaching can help medical students acquire and retain these skills [9-12].

Thus, the development of strategies targeting at strengthening communication has become a central topic in clinical and social research. About one decade ago, the theories and methodologies of doctor-patient communication skills training (CST) had been introduced in China. For instance, the Calgary-Cambridge Guide [13] and the SEGUE Framework [14] had been modified to conform to the Chinese culture. In 2003, the educators of Nanjing Medical University developed and deployed the first doctor-patient communication curriculum. Nonetheless, recent research [2] shows that only $40 \%$ of Chinese medical schools included doctor-patient communication in their curriculum, and most of the CST was an optional course. Furthermore, it is hard for residents and practicing physicians to receive CST continuing education. Compared to the identified substantial need for doctorpatient CST, the resources and endeavor devoted to it remain inadequate. As was suggested in Tu et's [15] investigation on outpatient communication in a large hospital in China, there is an extreme inequality of power between Chinese doctors and patients at the individual level. More public education and professional skills training are needed to improve the communication and promote mutual understanding between patients and doctors.

In addition to CST courses, simulated patients (SPs) are an important didactic tool in the education of medical students [16]. Medical curricula throughout the world consist of such simulation trainings [17-19]. In spite of this, Bokken L [20] pointed that there appear to be no clear standards with regard to effective feedback training for SPs. Moreover, there is no solid scientific basis in terms of the processes by which feedback is provided by SPs and the selection of domain(s) in which SPs give feedback often.

Recently, some researchers $[21,22]$ have reported that 3D printed models could improve the patients' understanding and compliance in surgery. However, some problems have also been identified, for example, this method is only suitable for some communication scenarios, and it consumes financial and material resources, and does not have the feasibility of wide application.

Therefore, in order to popularize the doctor-patient communication education and make learning methods more diversified, we try to combine online learning with communication skills learning. Under the circumstance, a new doctor-patient communication learning software is designed and produced in the paper. Meanwhile, its effectiveness has also been tested. It is a new method that was by no means available from domestic and overseas literatures $[12,23]$.

\section{Methods \\ Design and preparation of new doctor-patient communication learning software}

The doctor-patient communication learning software was written in the $\mathrm{C}++$ language, the development process of which was divided into three sequential stages: business modeling requirements phase, design phase and implementation phase of the project. The backend database involved the open source mysql, and the front end was rendered with Hyper Text Markup Language 5 technology. With its background supported by the Thinkphp framework for Model-View-Controller, the site was built on Sina cloud to facilitate program monitoring and subsequent migration. The basic information and answer records of all users were stored in the website database so that further data analysis could be carried out. The doctor-patient communication learning software was designed with the aim of transforming the traditional model of communication skills training for Chinese medical students in hopes that its rich and colorful forms could shed light on doctor-patient communication training program provided by the following link: http://yhgt.applinzi.com/.

The software mainly consists of two modules, namely a random module and a scenario module. The random module teaches students theoretical knowledge of doctor-patient communication skills, while the scenario module allows the learners to apply the knowledge gained in the random module to real case studies. There are some theoretical questions with 4 or 5 choices for students to choose in random module. Each question has one or more correct answers. As far as the scenario module is concerned, however, the questions from clinical cases are more practical, and each choice has a corresponding score, which means there are no absolutely correct answers. Once the students completed the test questions in the scenario module, the updated questionnaires were generated based on the answers (The example is shown in Fig. 1). Meanwhile, related questionnaires were emailed to friends or family who could play the role of the patient.

In clinical practice, SPs technology [24] is extensively applied in teaching and evaluation as SPs are able to provide credible interactions for students. However, without standardized training, friends or family as simulated patients are likely to be more real, effective and 


\section{a}

A middle-aged woman was diagnosed with ovarian cancer. She very worried about her condition and can't understanf Why she is in poor health. More than that, she even wouldn't know how to go on. To be a gynecologist, how will you communicate with the patient.

A. uterine fibroids is a common gynecological disease which can be solved by surgy. So don't worry about it.

B. You need to do surgery as soon. If the pathological diagnosis showed malignant, you may receive adjuvant radio (chemo-) therapy, extended resection,

C. This is so cruel to you. You should learn to strong.

D. Are you worried about your illness? Uterine fibroids are the most common benign tumors in women, which can be solved by surgy.Postoperative recovery was fast, does not affect the life and work.

\section{$\mathrm{b}$}

If you are a middle-aged woman, was diagnosed with ovarian cancer. You very worried about her your condition and can't understanf Why you are in poor health. More than that, you even wouldn't know how to go on. Are you satisfied with an explaination as follows, When talk to a doctor.

Uterine fibroids is a common gynecological disease which can be solved by surgy. So don't worry about it.

discontent

acceptable

content

Fig. 1 An example of scenario question and related question. If one chooses A in 1a, then the updated question accordingly is what one observes in $1 \mathrm{~b}$

closer to the actual situations. In this sense, the evaluation was more objective and sufficient.

All clinic cases in the scenario module were collected at the First Affiliated Hospital of Xi'an Jiaotong University from January 2015 and August 2015. Referencing more than 20 books and research articles about communication and medical ethics, our team members designed the test questions, which were then reviewed and revised by training experts from First Affiliated Hospital of Xi'an Jiaotong University.

\section{Participants and groups}

The study protocol was approved by the Ethics Committee of the First Affiliated Hospital of Xi'an Jiaotong University in China, and the research was conducted in the Gynecology and Obstetrics Department from 2015 to 2016. The design involved a selfcontrolled trial with one group. One software developer and six gynecologists (chief physician, associate chief physician, postgraduate [1:1:4]) participated in the research.

A total of 183 interns (79 males and 104 females, 4 ethnic minorities) from the First Affiliated Hospital of Xi'an Jiaotong University were selected as the research subjects whom were randomly divided into two groups. Of these
183 students, 10 missed the base value test and were excluded from analysis.

\section{Research protocol}

Self-controlled trial: The students' basic levels of doctorpatient communication were tested through the "doctorpatient communication scale" in May 2015; students who completed the doctor-patient communication skills training through the "doctor-patient communication software" were measured again in December 2015.

The SEGUE framework [14] was chosen as the "doctor-patient communication scale".

\section{Measures and statistical analyses}

The data analysis and statistics were performed with Statistical Program for Social Sciences (SPSS) software, version 10.0. A comparison between the pre-test and post-test scores was performed through the independent-sample $t$ test. Statistical significance was accepted at $P<0.05$.

\section{Results}

New doctor-patient communication learning software More than 20 professional books were consulted about doctor-patient communication, medical ethics and other 
subjects. Based on this, 150 theoretical questions (including regulations, skills, principles, etc.) were formulated according to relevant knowledge, and 100 questions were selected including 85 single choice questions and 15 multiple choice questions. More than 50 clinical cases were collected from internal medical departments (Department of Cardiology, Respiratory Medicine, Gastroenterology), Surgery Departments (Department of Urology, Department of Orthopedics, Department of General Surgery), Department of Obstetrics and Gynecology, Department of Pediatrics and other disciplines; 120 test questions and patient satisfaction questions were created from parts of 30 of the 50 clinical cases.

According to our design idea (as described in materials and methods), it took as long as two months to complete the original doctor-patient communication learning software. Two modules and an evaluation system are available in this software: random pattern, scenario pattern and a patient satisfaction questionnaire.

Random module (Fig. 2): There were 100 doctorpatient communication learning questions. Each time, 25 questions were randomly prepared in the system and a standard answer was set for students. After completing the exercise, students could view the results and repeatedly practice.

Scenario module (Fig. 3): In this module, there were 4 sets of tests involving the theoretic and scenario questions. An updated score was set accordingly to each answer; no standard answer was set in the scenario questions.

Patient satisfaction Questionnaire: Once the students completed the test questions, the related questionnaires were generated according to the answers. Students could not only invite patients to answer the questionnaire but also send the questionnaire to their relatives and friends by e-mail to answer it from the patient's point of view. It is an excellent assessment for the scenario module.

\section{Self-controlled trial (Table 1)}

For the first trial, a total of 183 questionnaires were issued, and 173 were actually recovered, at a return rate of 94.5\%; 183 questionnaires of second trial were issued, and 183 were actually recovered, with a return rate of

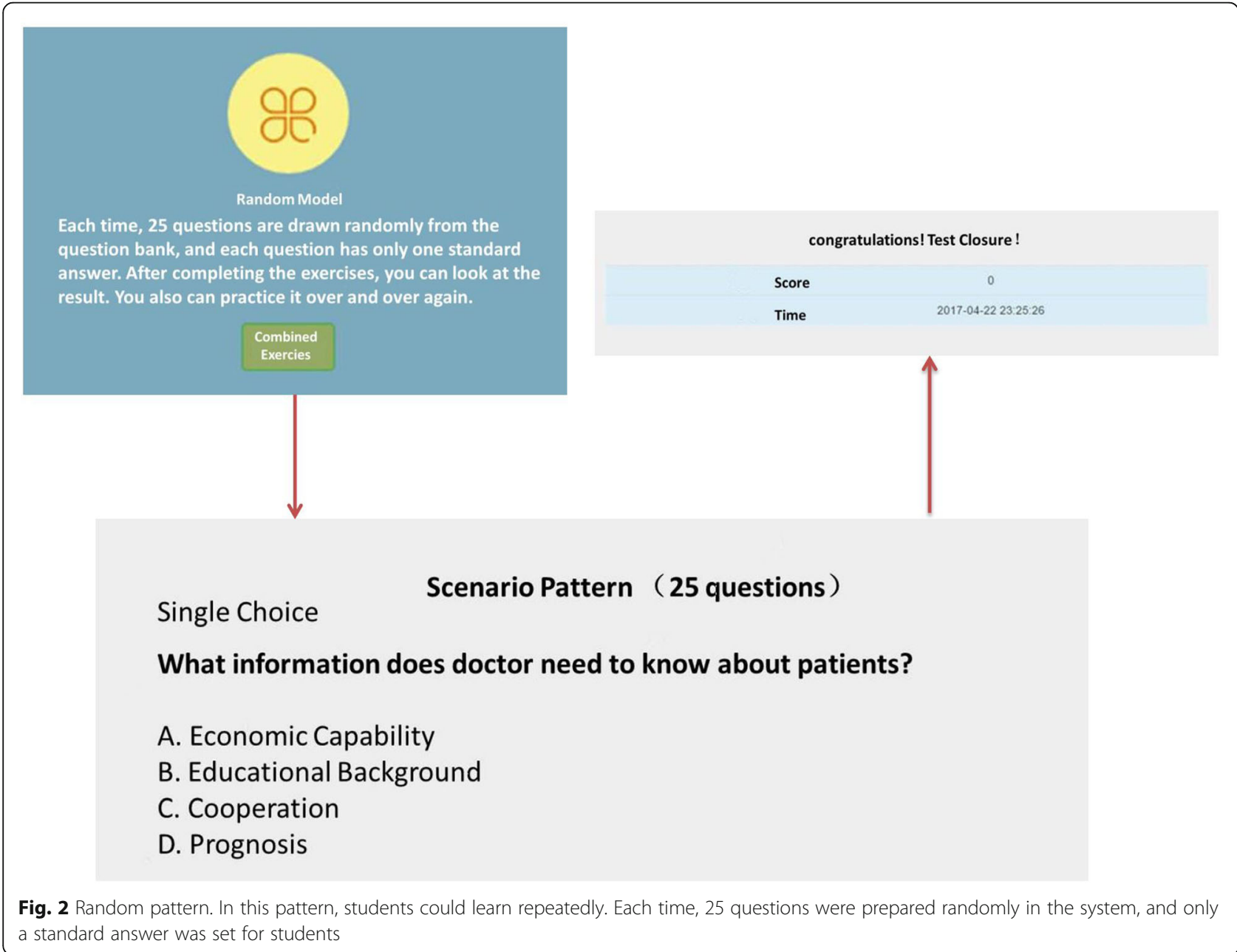




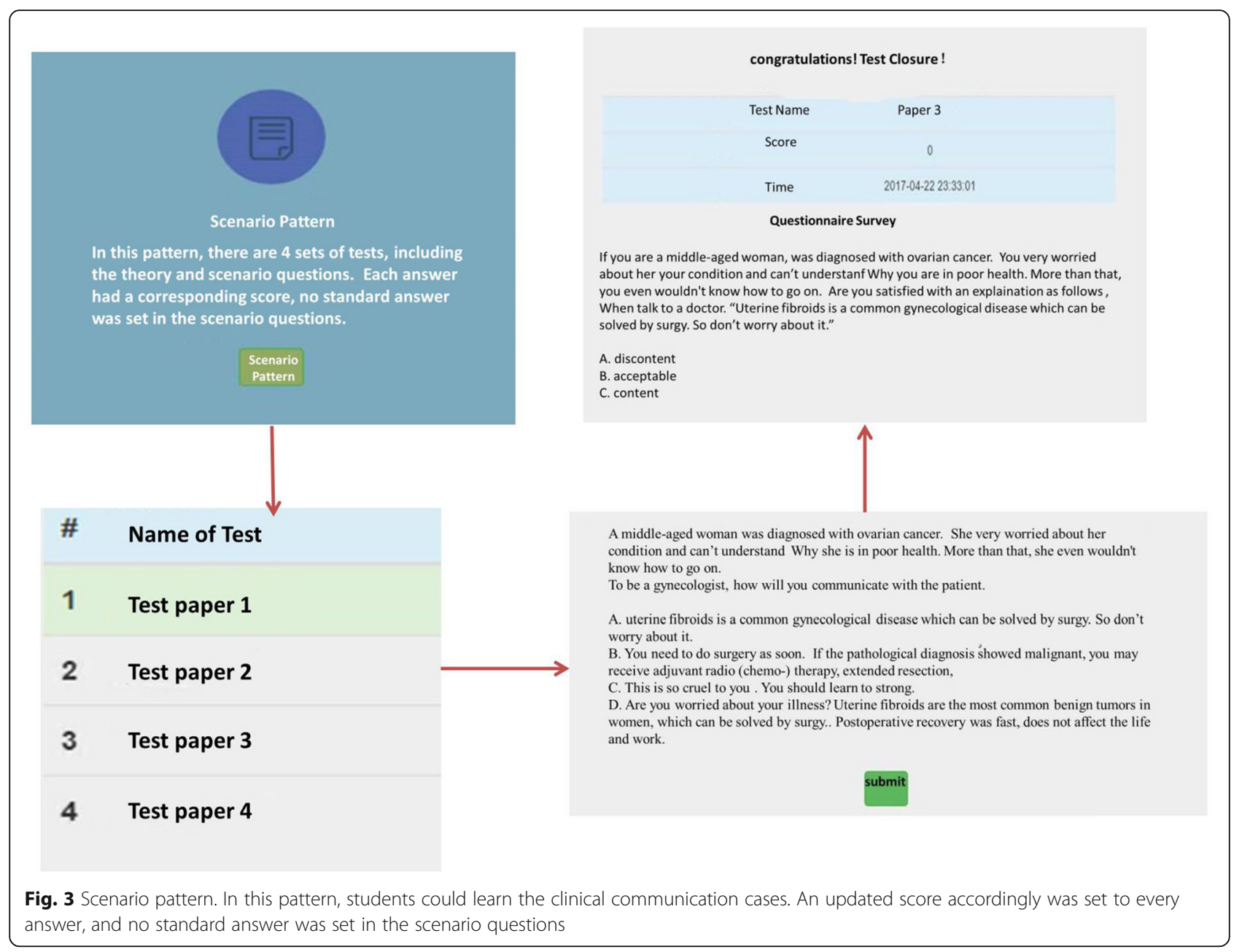

100\%. All the 183 interns completed the doctor-patient communication software learning.

Of the 183 students involved, 10 did not or not fully participate in the course. Thus, data sets of $N=173 \mathrm{stu}-$ dents (75 males and 98 females, 4 ethnic minorities) were available for analysis. The mean age of participants was 21.5 years $( \pm 2.40)$.

In order to judge the ability and function of the software in improving the communication level, 25 communication skills of SEGUE were tested by $\mathrm{t}$ test before and after learning. Seven results became significant. Specifically, they are the Courtesy title, Avoid inducing questions, Give patients time to express, Inquiring about healthy living, Talking about healthy living, Informing physical condition and Expressing concern.

However, through the study of the software, there was no significant improvement in most communication skills $(18 / 25)$ before and after learning. These skills are listed as follows: Inquiring about reasons for interrogation, Introduce operation process, Build trust relationships, Protect patients' privacy, Guide patients to talk about their symptoms, Systematic inquiry for Disease, Discuss about diagnosis and treatment, Quality of life, Give patients time to express, Listen carefully and give feedback, Verify patient information, Interpretation basis, Comfort and encourage patients, Adjust the way of interpretation, Identify patients' efforts, Observe the patients' suggestion, Maintain respectful tone, Make sure the patients have no problem, and Describe the treatment plan.

\section{Preparation stage}

The differences in courtesy with patients were statistically significant $(90.17 \%$ vs. $97.81 \% ; P=0.002)$. Other communication before the performance showed no significant difference in the two assessment results.

\section{Information collection}

In the collection process, compared to the first assessment results, more interns were able to pay attention to the social, psychological or emotional factors associated with disease $(37.16 \%$ vs. $10.98 \% ; P<0.001)$ and to 
Table 1 Statistical Reports about Controlled Trail

\begin{tabular}{|c|c|c|c|c|}
\hline & Base score & Test score & The value of $X^{2}$ & The value of $P$ \\
\hline The number of students & 173 & 183 & & \\
\hline \multicolumn{5}{|l|}{ Preparation Stage } \\
\hline courtesy title & $156(90.17)$ & $179(97.81)$ & 9.353 & 0.002 \\
\hline Inquiring about reasons for interrogation & $147(84.97)$ & $151(82.51)$ & 0.394 & 0.530 \\
\hline Introduce operation process & $17(9.83)$ & $23(12.57)$ & 0.670 & 0.413 \\
\hline Build trust relationships & $30(17.34)$ & $32(17.49)$ & 0.001 & 0.971 \\
\hline Protect patient privacy & $58(33.53)$ & $62(33.88)$ & 0.005 & 0.944 \\
\hline \multicolumn{5}{|l|}{ Information Collection } \\
\hline To guide the patients to talk about & $20(11.56)$ & $28(15.30)$ & 1.066 & 0.302 \\
\hline Systematic inquiry for Disease & $154(89.02)$ & $158(86.34)$ & 0.589 & 0.443 \\
\hline Asking about psychological factors & 19 (10.98) & $68(37.16)$ & 32.997 & $<0.001$ \\
\hline Discuss the diagnosis and treatment & $150(86.71)$ & $147(80.33)$ & 2.616 & 0.106 \\
\hline Quality of life & $56(32.37)$ & $57(31.15)$ & 0.061 & 0.804 \\
\hline Talking about healthy living & $18(10.40)$ & $78(42.62)$ & 46.871 & $<0.001$ \\
\hline Avoid inducing questions & $57(32.95)$ & $34(18.58)$ & 9.649 & 0.002 \\
\hline Give patients time to express & $53(30.64)$ & $77(42.08)$ & 5.021 & 0.025 \\
\hline Listen carefully and give feedback & $28(16.18)$ & $35(19.13)$ & 0.528 & 0.467 \\
\hline Verify patient information & $71(41.04)$ & $89(48.63)$ & 2.072 & 0.150 \\
\hline \multicolumn{5}{|l|}{ Information Given } \\
\hline Interpretation basis & $26(15.03)$ & $37(20.22)$ & 1.644 & 0.200 \\
\hline Informing physical condition & $15(8.67)$ & $72(39.34)$ & 45.312 & $<0.001$ \\
\hline Comfort and encourage patients & $11(6.34)$ & $18(9.84)$ & 1.437 & 0.231 \\
\hline Adjust the way of interpretation & $55(31.79)$ & $70(38.25)$ & 1.629 & 0.202 \\
\hline \multicolumn{5}{|l|}{ Patients Understanding } \\
\hline Identify patient efforts & $57(32.95)$ & $55(30.05)$ & 0.345 & 0.557 \\
\hline Observe the patient's suggestion & $35(20.23)$ & $53(28.96)$ & 3.643 & 0.056 \\
\hline Expressing concern & $46(26.59)$ & $97(53.01)$ & 25.820 & $<0.001$ \\
\hline Maintain respectful tone & $161(93.06)$ & $162(88.52)$ & 2.178 & 0.140 \\
\hline \multicolumn{5}{|l|}{ Inquisition Ending } \\
\hline Make sure the patient has no problem & $49(28.32)$ & $52(28.42)$ & 0.069 & 0.793 \\
\hline Describe the treatment plan & $57(32.95)$ & $55(30.05)$ & 0.345 & 0.557 \\
\hline
\end{tabular}

discuss healthy lifestyle or disease prevention measures with patients $(42.62 \%$ vs. $10.40 \% ; P<0.001)$. The results of the other projects were not statistically significant during the two-evaluation process.

\section{Information given}

Only a small number of interns were able to provide adequate patient information during doctor-patient communication. Of these, after completing the study, more interns were able to inform patients of their current physical condition $(39.34 \%$, vs. $8.67 \%, P<0.001)$, including physical, laboratory, and diagnostic results. The other results were not statistically significant in the twoevaluation process.

\section{Inquisition ending}

There was no significant difference between the two assessments.

\section{Discussion}

Network has brought dramatic changes to our life and added richness to learning styles [25-27]. Although the traditional teaching method in doctor-patient communication skills training was prevalent in medical schools and hospitals in China, its practicality has been challenged by the worsening doctor-patient relationship [7, 21, 22]. As the information technology advances, computer-aided learning has emerged and gained popularity as a complementary approach in education and training due to its 
convenience and interactivity [28-30]. Consequently, the study develops the doctor-patient communication learning software to create an effective and interactive learning environment for interns. In addition, the accompanying patient satisfaction questionnaire can combine course study with clinical practice. A comparison is made about the effect of our software on students' learning and communication skill improvement between the pre-training and post-training sessions in the study.

According to the results, when obtaining information from patients, more interns allowed the patients sufficient time to describe their symptoms and share their perspectives. With the training and feedback from simulated patients (friends and family to play the role of patient), some students can recognize their deficiencies and pay more attention to patients gradually. Furthermore, the interns in this study also have more experiences in demonstrating medical care, concern, empathy and compassion, as well as the ability to communicate information effectively with patients. However, it is not indicated in the study that the interns significantly improved their ability to explain the disease more professionally because the relevant theoretical knowledge was not included in the software. Moreover, in terms of most of the learning and improvement of communication skills, the software does not show its superiority. It may be related to the monotonous settings of software problems, inadequate learning content and insufficient diversity of learning forms.

Medicine involves the integration of not only art and science but also magic and creative ability, and the building of a harmonious doctor-patient relationship reflects such artistic quality [8, 31, 32]. Communication and interpersonal skills for doctors, in particular young Chinese medical students, plays a big role. We hope that the application of this doctor-patient communication learning software turns out helpful and valuable.

The study was limited because of the use of fewer test questions and inadequate variety of the subjects. Some insufficiencies can also be found in the current study. Specifically, we plan to improve and refine our explorations along the following directions: First, establishment of an independent application; Second, addition and modification of questions; Third, addition of new learning patterns; Fourth, extending the user population (fresh doctors, nurses, and patients). Besides, in the preliminary application, only the self-controlled trial was made. In the subsequent study, a paired designed trial is expected to prepare to evaluate the software more fully based on current practices.

\section{Conclusions}

We designed a type of learning software to train interns to advance their communication skills and evaluated its validity for improving doctor-patient communication in self-controlled trials. The self-controlled tests were implemented among 183 interns to assess the quality of communication skill acquisition by applying the new learning software. It is concluded that the doctor-patient communication software can provide a practice platform for interns. In the part of Patient Satisfaction Questionnaire, interns can acquire some feedback from patients, which allows them to know more about doctor-patient communication skills.

Project name: Teaching Reform Project of the First Affiliated Hospital of Xi'an Jiaotong University.

Project home page: http://yhgt.applinzi.com/

Operating system(s): Linux.

Programming language: PHP language.

Other requirements: Nginx 1.6.3 or higher, Thinkphp 3.2.3 or higher.

Any restrictions to use by non-academics: licence needed.

\section{Abbreviations \\ CST: Communication skills training; SEGUE: Set Elicit Give Understand End; SPs: Simulated patients; SPSS: Statistical Program for Social Sciences}

\section{Acknowledgements}

The authors in this manuscript want to thank Prof. Yunjing Zhang in the First Affiliated Hospital of Xi'an Jiaotong University for her constructive suggestion.

\section{Authors' contributions}

QL conceived the original idea, coordinated the research and is responsible for the final version. CS analyzed statistical data and drafted the manuscript. CS and JZ gathered and analyzed qualitative data. LZ and QW collaborated in the interpretation of quantitative data. SZ wrote and validated the software. QU and QS helped revising the manuscript. All authors participated in the critical version of the manuscript and approved the final version.

\section{Funding}

This investigation was supported, in part, by the Clinical Education Funds of 1st Affiliated Hospital of XJTU (Grant no.14YB16; Grant no.15ZD10). The funding body played no role in the design of the study and collection, analysis, and interpretation of data and in writing the manuscript.

\section{Availability of data and materials}

The data is presented in the table and figures. Readers seeking access to any of the raw data, software code and test framework may contact the corresponding author, Qiling Li: liqiling@mail.xjtu.edu.cn

License: This article is published under license to BioMed Central Ltd. This is an Open Access article distributed under the terms of the GNU General Public License (http://www.gnu.org/licenses/old-licenses/gpl-2.0.html), which permits unrestricted use, distribution, and reproduction in any medium, provided the original work is properly cited.

\section{Ethics approval and consent to participate}

The First Affiliated Hospital ethics committee (Xi'an, Shaanxi) reviewed the research proposal and granted insurance. Written informed consent was obtained from all individual participants included in the study.

Consent for publication

Not applicable.

Competing interests

The authors declare that they have no competing interest. 


\section{Author details}

${ }^{1}$ First Affiliated Hospital, Xi'an Jiaotong University, 277 Yanta West Road, Xi'an, Shaanxi, China. ${ }^{2}$ Affiliated Tumor Hospital, Xinjiang Medical University, 789 Suzhou East Street, Urumqi, Xinjiang, China. ${ }^{3}$ Department of Electronic Engineering, Xidian University, 2 Taibai South Road, Xi'an, Shaanxi, China. ${ }^{4}$ Cardiovascular Research Institute, Morehouse School of Medicine, 720 Westview Drive, Atlanta, Gorge, USA.

Received: 12 May 2019 Accepted: 22 December 2019

Published online: 08 January 2020

\section{References}

1. Zeng J, Zeng XX, Tu Q. A gloomy future for medical students in China. Lancet. 2013;382:1878.

2. Liu X, Rohrer W, Luo A, Fang Z, He T, et al. Doctor-patient communication skills training in mainland China: a systematic review of the literature. Patient Educ Couns. 2015;98:3-14.

3. Fritzsche K, Scheib P, Ko N, Wirsching M, Kuhnert A, et al. Results of a psychosomatic training program in China, Vietnam and Laos: successful cross-cultural transfer of a postgraduate training program for medical doctors. Biopsychosoc Med. 2012;6:17.

4. Jagosh J, Donald Boudreau J, Steinert Y, Macdonald ME, Ingram L. The importance of physician listening from the patients' perspective: enhancing diagnosis, healing, and the doctor-patient relationship. Patient Educ Couns. 2011:85:369-74.

5. Wuensch A, Tang L, Goelz T, Zhang Y, Stubenrauch S, et al. Breaking bad news in China--the dilemma of patients' autonomy and traditional norms. A first communication skills training for Chinese oncologists and caretakers. Psychooncology. 2013;22:1192-5.

6. Aspegren K. BEME guide no. 2: teaching and learning communication skills in medicine-a review with quality grading of articles. Med Teach. 1999;21:563-70.

7. Aitini $E$, Martignoni $G$, Labianca R, Italian Group for the study of Digestive Tract C. Communication models for doctor-patient relationships. J Cancer Educ. 2014;29:211-2.

8. Ha JF, Longnecker N. Doctor-patient communication: a review. Ochsner J. 2010;10:38-43.

9. Zimmermann C, del Piccolo L, Mazzi MA. Patient cues and medical interviewing in general practice: examples of the application of sequential analysis. Epidemiol Psichiatr Soc. 2003;12:115-23.

10. Hu W, Song Y, Zhong X, Feng J, Wang P, et al. Improving doctor-patient communication: content validity examination of a novel urinary systemsimulating physical model. Patient Prefer Adherence. 2016;10:2519-29.

11. McAlinden $\mathrm{C}$. The importance of doctor-patient communication. Br J Hosp Med (Lond). 2014;75:64-5.

12. Fillon M. New tool improves doctor-patient communication. J Natl Cancer Inst. 2015;107(4).

13. Parry RH, Brown K. Teaching and learning communication skills in physiotherapy: what is done and how should it be done? Physiotherapy. 2009;95:294-301.

14. Makoul G. The SEGUE framework for teaching and assessing communication skills. Patient Educ Couns. 2001;45:23-34.

15. Tu J, Kang G, Zhong J, Cheng Y. Outpatient communication patterns in a cancer hospital in China: a qualitative study of doctor-patient encounters. Health Expect. 2019;22(3):594-603.

16. Jabeen D. Use of simulated patients for assessment of communication skills in undergraduate medical education in obstetrics and gynaecology. J Coll Physicians Surg Pak. 2013;23:16-9.

17. Woodward CA, Neufeld VR, Norman GR, Stillman PL. Symposium: simulated patients in evaluation of medical education and practice. Proc Annu Conf Res Med Educ. 1983;22:238-44.

18. Nestel D, Clark S, Tabak D, Ashwell V, Muir E, et al. Defining responsibilities of simulated patients in medical education. Simul Healthc. 2010;5:161-8.

19. Cleland JA, Abe K, Rethans JJ. The use of simulated patients in medical education: AMEE guide no 42. Med Teach. 2009;31:477-86.

20. Bokken L, Linssen T, Scherpbier A, van der Vleuten C, Rethans JJ. Feedback by simulated patients in undergraduate medical education: a systematic review of the literature. Med Educ. 2009;43:202-10.

21. Lou Y, Cai L, Wang C, Tang Q, Pan T, et al. Comparison of traditional surgery and surgery assisted by three dimensional printing technology in the treatment of tibial plateau fractures. Int Orthop. 2017;41:1875-80.
22. Zheng W, Chen C, Zhang C, Tao Z, Cai L. The feasibility of 3D printing technology on the treatment of Pilon fracture and its effect on doctorpatient communication. Biomed Res Int. 2018;2018:8054698.

23. Kashgary A, Alsolaimani R, Mosli M, Faraj S. The role of mobile devices in doctor-patient communication: a systematic review and meta-analysis. J Telemed Telecare. 2017;23(8).

24. Murphy S, Imam B, Maclntyre DL. Standardized patients versus volunteer patients for physical therapy Students' interviewing practice: a pilot study. Physiother Can. 2015;67:378-84.

25. Marden NY, Ulman LG, Wilson FS, Velan GM. Online feedback assessments in physiology: effects on students' learning experiences and outcomes. Adv Physiol Educ. 2013;37:192-200.

26. Slade JD, Robb M, Sherrod B, Hunker D. Online adjunct faculty support: an innovative use of a learning management system. Nurse Educ. 2017;42(3).

27. Stafford T, Haasnoot E (2016) Testing sleep consolidation in skill learning: a field study using an online game. Top Cogn Sci.

28. Ellman MS, Schwartz ML. Online Learning Tools as Supplements for Basic and Clinical Science Education. J Med Educ Curric Dev. 2016;3.

29. Kimiafar K, Sarbaz M, Sheikhtaheri A. Online survey software as a data collection tool for medical education: a case study on lesson plan assessment. Med J Islam Repub Iran. 2016;30:464.

30. King C, Robinson A, Vickers J. Online education: targeted MOOC captivates students. Nature. 2014;505:26.

31. Ong LM, de Haes JC, Hoos AM, Lammes FB. Doctor-patient communication: a review of the literature. Soc Sci Med. 1995;40:903-18.

32. Williams S, Weinman J, Dale J. Doctor-patient communication and patient satisfaction: a review. Fam Pract. 1998;15:480-92.

\section{Publisher's Note}

Springer Nature remains neutral with regard to jurisdictional claims in published maps and institutional affiliations.
Ready to submit your research? Choose BMC and benefit from:

- fast, convenient online submission

- thorough peer review by experienced researchers in your field

- rapid publication on acceptance

- support for research data, including large and complex data types

- gold Open Access which fosters wider collaboration and increased citations

- maximum visibility for your research: over $100 \mathrm{M}$ website views per year

At BMC, research is always in progress.

Learn more biomedcentral.com/submissions 